\title{
Sistemas de GeSTÃo da InOVAÇÃo e TranSFORMAÇão Digital: EM BUSCA DE UMA ABORDAGEM INTEGRADA
}

Gustavo Pereira Franco (gpframco@gmail.com) - Universidade Federal de Minas Gerais (UFMG)

Leonardo Duarte Martins (leonardodm@gmail.com) - Universidade Federal de Minas Gerais (UFMG)

Raphaela Cristina Severiano Athanazio (raphaathanazio@ufmg.br) - Universidade Federal de Minas Gerais (UFMG)

Ana Carolina dos Santos Paes (anacarolinads.paes@gmail.com) - Universidade Federal de Minas Gerais (UFMG)

Raoni Barros Bagno (ㅎbagno@gmail.com) - Universidade Federal de Minas Gerais (UFMG)

\section{RESUMO}

Sistemas de Gestão da Inovação (SGI) têm sido debatidos há muitos anos, tendo em vista a importância central para as empresas de inovar regularmente. Mais recentemente, temos testemunhado o rápido crescimento do debate sobre a Transformação Digital (TD), que pode ser descrita como um processo de mudança organizacional, no qual as tecnologias digitais desempenham papel central. Sendo fortemente transversal em termos de conhecimento e impactos gerados, o debate da TD tem emergido de forma bastante distribuída entre várias vertentes trazendo riqueza de perspectivas, mas também complexidade. As potencialidades de uma visão mais integrada entre SGI e TD são significativas: por um lado, um SGI pode nortear iniciativas de TD por meio de estrutura e processos concebidos para lidar com projetos de alta incerteza; por outro, a TD traz uma série de conceitos e tendências, tecnológicos e organizacionais, que podem fomentar SGIs aprimorados, que melhor se amoldem às novas dinâmicas de negócio. Contudo, tal integração ainda se mostra bastante incipiente e os campos seguem majoritariamente trajetórias paralelas. Assim, por meio de revisão bibliográfica e análise conceitual, este estudo identifica pontos de intercessão entre SGI e a TD e investiga como elementos dessas abordagens podem se apoiar mutuamente.

Palavras chave: Transformação Digital; Sistema de Gestão da Inovação; Inovação Digital; tecnologias

digitais. 


\section{INTRODUÇÃO}

Inovar tornou-se uma questão essencial para uma atuação de sucesso no mercado e é um fator chave para o desenvolvimento econômico e competitivo para empresas, regiões e nações (FrAmbaCH; SCHILlEWAERT, 2002). Segundo Crossan e Apaydin (2010), inovação consiste na produção ou adoção, assimilação e exploração de uma novidade nas esferas econômica e social; renovação e ampliação de produtos, serviços e mercados; desenvolvimento de novos métodos de produção; ou estabelecimento de novos sistemas de gerenciamento. Assim, para promover a inovação de forma regular e contínua nas organizações, formas e abordagens para Sistemas de Gestão da Inovação (SGI) têm sido propostos na literatura e na prática. O'Connor et al. (2008) definem um sistema de gestão como um conjunto de elementos necessários para fazer uma organização funcionar efetiva e eficientemente. Assim, o sistema de gestão da inovação serve como norteador para gerentes de inovação organizarem e gerenciarem atividades e responsabilidades no entorno do processo de inovação.

Em paralelo, a difusão de tecnologias digitais tem acelerado a transição, não somente de uso e aplicação de tecnologias, mas também de diversos valores, aspectos organizacionais e comportamentais da sociedade. Nos últimos anos, a Transformação Digital (TD) tem emergido como um poderoso propulsor da inovação, capaz de melhorar processos, modelos de negócios e causar outras mudanças nas organizações. O debate a respeito desse fenômeno apresenta um caráter fortemente transversal, uma vez que diversas escolas de pensamento apresentam diferentes teorias e conceitos sobre o tema (RIASANOW et al., 2019). Vial (2019) descreve a TD como um processo que objetiva melhorar uma entidade, desencadeando mudanças significativas por meio de combinações entre tecnologias de computação, comunicação e de conectividade. Já Hanelt et al. (2020) enfatizam o caráter organizacional da transformação digital, definindo-a como uma mudança organizacional que é desencadeada e moldada pela difusão das tecnologias digitais.

Muito embora a intercessão entre gestão da inovação (e sistemas de gestão que a promovem) e as demandas e oportunidades no campo da TD pareçam ser evidentes, dois desafios emergem deste contexto: (i) aparentemente, debates na literatura sobre gestão da inovação e sobre transformação digital tem percorrido trajetórias distintas, com poucos pontos de contato. Como consequência, as contribuições da literatura, apesar de ricas, trazem desafios para interpretação e aplicação práticas em ambos os temas e perdem-se oportunidades de 
aprendizados mútuos e analogias no campo teórico; (ii) como um fenômeno complexo e abrangente, a transformação digital impacta empresas de diferentes formas e dimensões. Assim, presume-se que não possa ser contida por completo pelo debate de gestão da inovação e nem a conter adequadamente. $\mathrm{O}$ desvelamento das intercessões entre estes dois campos e suas contribuições mútuas torna-se essencial para se propor avanços. O objetivo desse estudo encontra-se nessa lacuna: identificar intercessões entre os campos da gestão da inovação e da TD por meio de revisões de literatura sobre esses temas e discutir as oportunidades de contribuições mútuas e desafios que emergem a partir destes pontos.

\section{METODOLOGIA}

O estudo da literatura utilizado para conduzir essa revisão foi feito de formas distintas para os dois campos considerados - TD e SGI. No campo da TD, após imersão inicial nas principais bases de dados (WoK, Scopus, Google Scholar), alguns pontos se tornaram claros: o crescimento exponencial desta literatura nos últimos anos, a pluralidade de estudos vindos de diferentes campos do conhecimento e a disponibilidade de um número considerável de revisões de literatura recentes (5 anos ou menos) vindos destes diferentes campos. A partir destas constatações, houve um redirecionamento dos esforços para, ao invés de se concentrarem num levantamento mais abrangente da literatura, se voltarem à identificação e priorização de revisões de literatura relevantes e recentes dos diferentes campos de estudo que tangenciam o fenômeno (Figura 1).

Para a busca dos textos utilizaram-se os termos "digital transformation" e "digital disruption" como um primeiro critério. Em seguida, adicionou-se a palavra-chave "Review" no tópico das buscas nas bases de dados e utilizou-se o JCR como critério de priorização. Onze revisões de literatura resultantes foram então estudadas em detalhe. Ao longo da elaboração deste artigo outros textos (normalmente citados pelas revisões priorizadas) foram sendo agregados para melhor elaboração dos argumentos.

No campo de SGI, a seleção de textos ocorreu essencialmente pelo método "bola de neve", priorizando-se artigos que tangenciassem a perspectiva de sistemas de gestão dentro do debate mais amplo de inovação sistemática a partir de trajetória já consolidada de pesquisa do grupo ao longo dos últimos 10 anos (e.g. BAGNO; LeIVA; OliveIRA, 2015; BAGNO; SALERNO; Silva, 2017; BAGNO et al., 2020). Este levantamento foi atualizado por busca complementar nas bases de dados utilizando-se no título os termos "innovation management syst*" e "innovation syst*”, neste último caso excluindo-se estudos focados em outros níveis de 
análise que não o da organização, principalmente aqueles oriundos da perspectiva econômica de sistemas nacionais ou regionais de inovação.

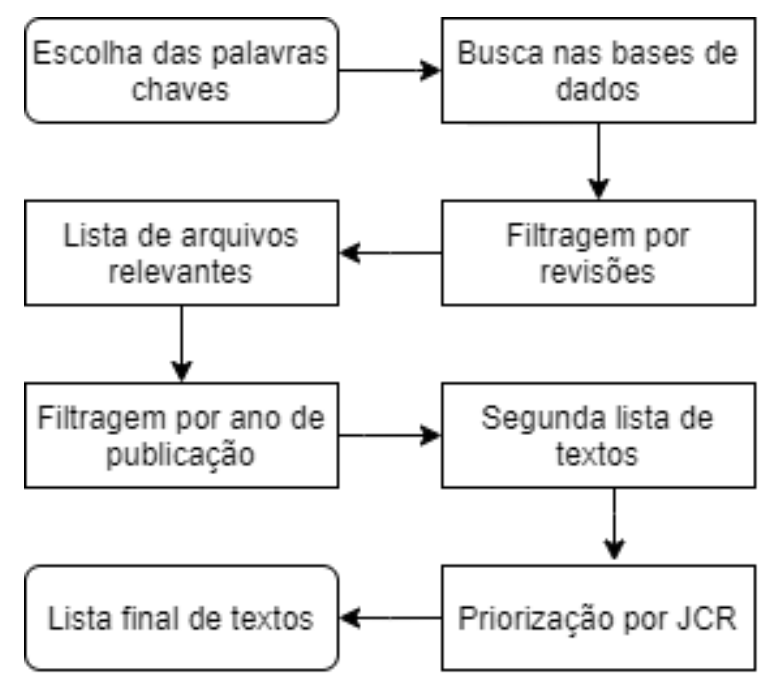

Figura 1 - Busca e seleção de revisões de literatura em Transformação Digital. Fonte: dos autores.

A identificação de elementos organizacionais comuns presentes nas sínteses realizadas em ambos os campos de estudo (TD e SGI) permitiram o confronto das contribuições de cada campo por categoria e a elaboração das reflexões desenvolvidas no tópico 4.

\section{REVISÃO TEÓRICA}

\subsection{Sistemas de Gestão da Inovação - SGI}

Van Lancker et al. (2016) definem o sistema de inovação organizacional como sendo uma rede inovadora de diversos atores, colaborando com uma organização focal inovadora em um processo para gerar, desenvolver e comercializar um novo conceito moldado pelas instituições. Destacam-se cinco componentes estruturais nesse sistema: atores, processo, portfólio, rede e instituições. Alguns desses aspectos se assemelham às dimensões propostas por O'Connor et al. (2008), que delimitam o sistema como sendo um conjunto de elementos necessários para fazer a organização funcionar de forma efetiva e eficiente: mandatos e responsabilidades, estrutura e processos, recursos e habilidades, liderança e governança, métricas e sistemas de recompensas (figura 2). Outras perspectivas presentes na literatura convergem com estes pontos em relação a seus elementos constituintes (e.g., ADAMs; Bessant; PhelPS, 2006; BAGNO; LeIVA; OliVeIRA, 2015; TIDD, 2021). 


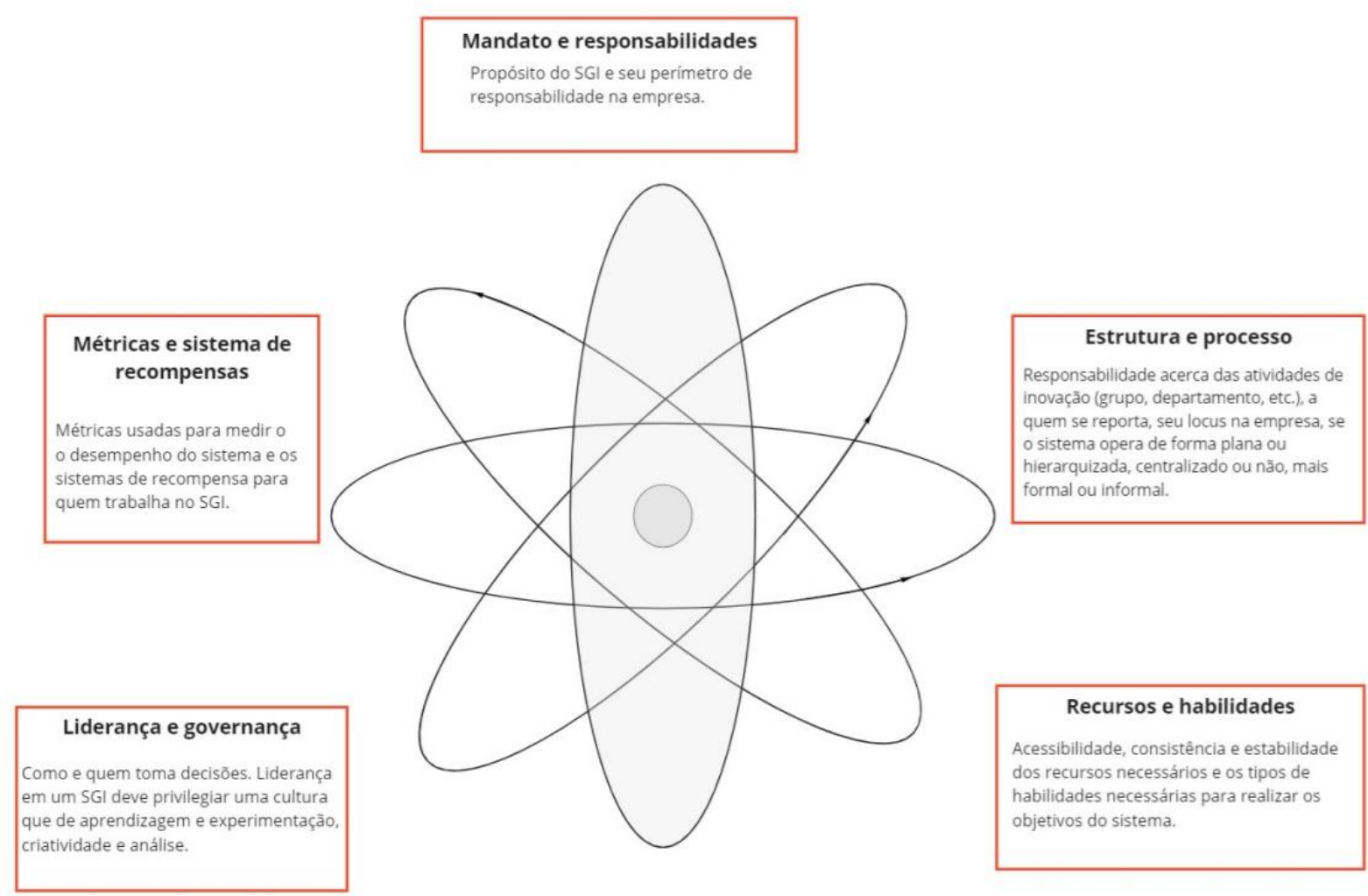

Figura 2 - Elementos do Sistema de Gestão da Inovação. Fonte: adaptado de O'Connor et al. (2008).

Em um SGI todos os elementos mencionados orbitam em torno de um processo de inovação (c.f. BAGNO; SAlerno; Silva, 2017). O entendimento da inovação como resultado de um processo levanta a necessidade de gerenciá-lo e visualizá-lo como uma cadeia de geração de valor (HANSEN; BIRKINSHAw, 2007). Porém, dados os riscos e incertezas que tipicamente caracterizam os projetos de inovação, trazem complexidade e desafios especiais a estes tipos de projetos como constantes contratempos, possíveis intervenções externas, multiplicidade e divergência de ideias, além de mudanças contínuas dos critérios de sucesso (TIDD, 2021). Por isso, Ries (2011) reforça a necessidade de que o processo de inovação seja flexível, iterativo e capaz de mudar de direção quando assim for necessário.

Alguns aspectos de ordem social nas organizações se destacam na busca de se tornar o processo de inovação algo recorrente nas empresas no contexto de um SGI. Tidd (2021) ressalta sete desses aspectos: visão compartilhada, liderança e vontade de inovar; estrutura apropriada; identificação e valorização de indivíduos-chave; trabalho de equipe eficaz; visão para inovação de alto desempenho; clima criativo e foco externo. A experiência técnica da liderança se torna uma influência para o desempenho dos times de inovação, ampliando a 
capacidade cognitiva, a resolução criativa de problemas e habilidades de processamento de informações (TIDD, 2021).

\subsection{Transformação Digital}

Vial (2019) descreve a TD como um processo de mudança organizacional, mas no qual as tecnologias digitais desempenham um papel central, o que reforça a dualidade organizaçãotecnologia (figura 3). Já Nadkarni e Prügl (2020) trazem uma distinção entre os elementos da Transformação Digital, classificando-os naqueles puramente tecnológicos e os tipicamente organizacionais. Estes são compostos por aspectos internos e do ambiente, que definem a organização e que desempenham determinados papéis, ou que precisam reconfigurar-se de alguma forma para viabilizar o processo de transformação. Complementarmente, Nambisan et al. (2017) propõem o conceito de inovação digital, como sendo a criação e consequentes mudanças na oferta de mercados e modelos ou processos de negócios que resultam da utilização das tecnologias digitais.

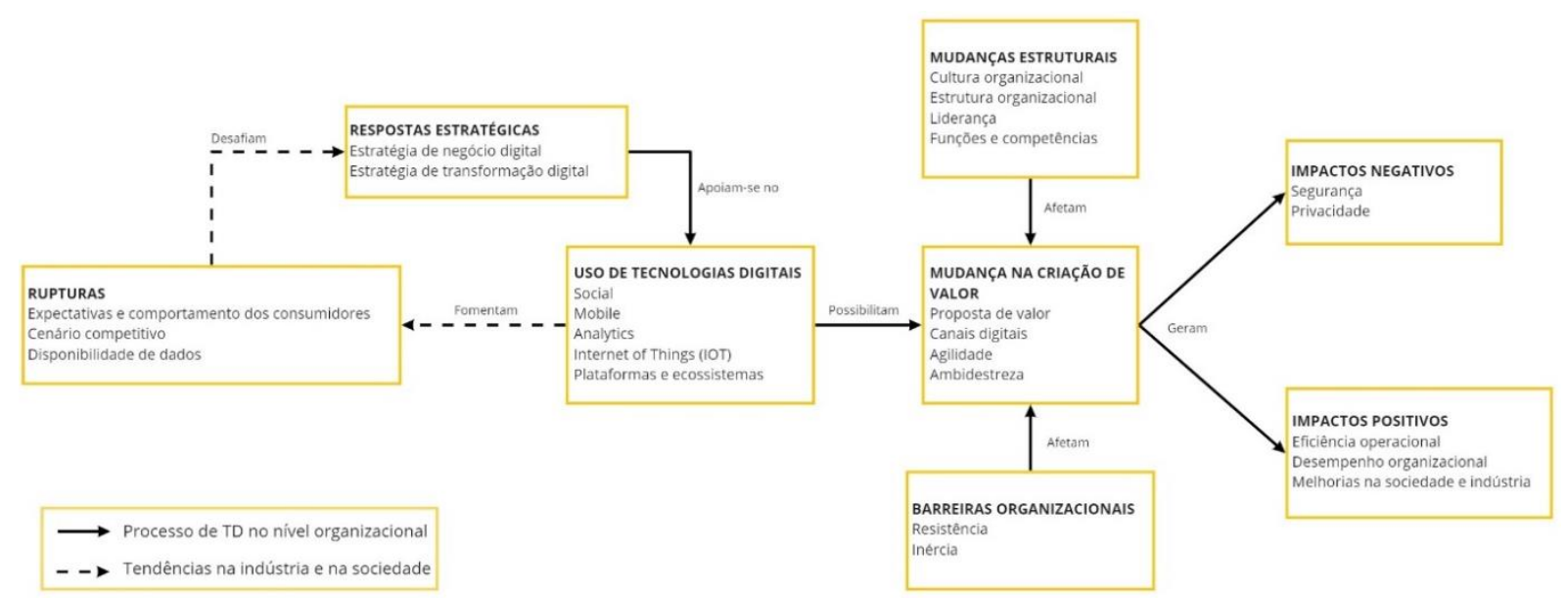

Figura 3 - Modelo conceitual de TD. Fonte: adaptado de Vial (2019).

Como elementos tecnológicos, Sebastian et al. (2017) classificam as tecnologias digitais sob o acrônimo SMACIT: Social, Mobile, Analytics, Cloud e IoT. Porém, a transformação digital não deve ser relacionada à utilização de tecnologias específicas, já que podem se tornar ultrapassadas no futuro (RIASANOW et al., 2019). Ainda assim, é importante compreender as tecnologias que estão sendo mais utilizadas no contexto de TD, uma vez que elas têm o potencial de catalisar transformações sociais, mudando setores econômicos, ritmo de trabalho e produção (BURRI, 2017). À medida que essas tecnologias fornecem mais informações, 
computação, comunicação e conectividade, elas permitem novas formas de colaboração entre redes distribuídas de diversos atores em direção a uma maior adaptabilidade ligada, principalmente, ao ambiente em que a empresa está inserida (VIAL, 2019; HANELT et al., 2020). Ao fazer isso, novas dependências entre empresas são criadas afetando indivíduos, indústrias e sociedade, e fomentando a criação de ecossistemas empresariais mais dinâmicos (KoPAlle; Kumar; Subramaniam, 2020).

Por fim, é importante considerar os elementos organizacionais que caracterizam um processo de TD, como: (i) liderança, que deve-se engajar em transparência e adaptabilidade ante aos desafios de transformação do fenômeno (DENNING, 2018; NADKARNI; PrÜGL, 2020); (ii) estrutura organizacional da empresa, que deve ser repensada a partir dos impactos causados pelas tecnologias digitais (GEBAYEW et al., 2018); (iii) capacidades de organização e gestão dadas pelo desenvolvimento de capacidades dinâmicas, além da combinação entre as capacidades analógicas e digitais (HANELT et al., 2020); (iv) cultura organizacional baseada em um pensamento inovador, aberta para promoção horizontal de parcerias externas, flexibilidade, multifuncionalidade das equipes e processos decisórios baseados em dados e nos clientes (BUVAT et al., 2017).

\section{DISCUSSÃO}

As possibilidades associadas à uma visão mais integrada entre TD e SGI são vastas. Observando a demanda por uma síntese dentro dos propósitos do presente texto, os subtópicos seguintes trazem os pontos considerados mais relevantes. Ao final, a figura 4 apresenta uma síntese visual do debate.

\subsection{Elementos organizacionais do Sistema de Gestão da Inovação convergentes com as demandas e desafios da Transformação Digital}

Um SGI é pautado sobre elementos e capacidades organizacionais que convergem com pilares necessários a um processo de TD, tais como liderança, cultura e estrutura organizacional. No caso da liderança, Van Lancker et al. (2016) destacam seu papel central como referência, suporte e legitimação para um processo de constante aprendizagem e interação entre grupos de trabalho (O'CONNOR et al., 2008; TIDD, 2021). Paralelo a isso, o líder deve valorizar a transparência e adaptação, promovendo um ambiente facilitador de futuras inovações e de 
mudanças culturais, como a inclusão de tecnologias digitais (MUMFORD; LICUANAN, 2004; DENNING, 2018).

Sobre cultura, uma vez que a TD exige um pensamento inovador, aberto e disruptivo que apoie a dinamicidade da tomada de riscos e a exploração de ideias (BUVAT et al., 2017; ROMERO et al., 2019; HANELT et al., 2020), a cultura inovadora fomentada por um SGI poderia estender sua abordagem para promover a chamada cultura digital. Ernst (2003) destaca que esse trabalho deve ser eficaz e multidisciplinar, apoiado por sistemas de motivação relevantes e métricas de desempenho (c.f. O'CONNOR et al., 2008; TIDD, 2021).

Por fim, o sistema de gestão deve estar suportado por uma estrutura organizacional que forneça liberdade para permitir a exploração de possibilidades criativas, mas também controle e disciplina para se gerenciar a inovação de forma efetiva (ADAMS; BESSANT; PHELPS, 2006). Este ponto se associa ao desenvolvimento de capacidades dinâmicas visando agilidade e adaptabilidade às condições do mercado (TEECE; PISAnO; Shuen, 1997; Mir; CASAdesús; PeTnJi, 2016). Associado a essas capacidades, Adams; Bessant e Phelps (2006) destacam também a necessidade de o SGI pavimentar ações para identificar, adquirir e utilizar conhecimento externo e capacidade de absorção de novos conhecimentos para colocá-los em prática para fins comerciais. No contexto de TD tais elementos são fundamentais no equilíbrio entre capacidades chamadas de "analógicas" e "digitais", e das capacidades de sensoriamento de uma empresa para implementar e operar em redes, plataformas e ecossistemas (NADKARNI; PRÜGL, 2020; VERHOEF et al., 2021). Hanelt et al. (2020) também ressaltam sua influência em estruturas organizacionais mais ágeis voltadas a mudança contínua juntamente com mecanismos de coordenação para assimilar tecnologias digitais dentro da organização.

Uma vez que a gestão da inovação contempla invariavelmente a consideração de fontes externas para impulsionar o processo de inovação (ANTHONY; JOHNSON; SINFIELD, 2008), é necessário promover uma rede extensa e dinâmica de colaborares e parceiros como formas de angariar recursos humanos e financeiros complementares (VAN LANCKER et al., 2016; TIDD, 2021), além de acesso a diferentes conhecimentos especializados. Essa rede de inovação baseada na colaboração vai de encontro ao contexto de ecossistemas empresariais proeminentes nos debates de TD e ao desenvolvimento do que Kopalle; Kumar e Subramaniam (2020) chamam de uma capacidade de rede digital. Esta visaria atrair, ligar e envolver um conjunto heterogêneo de atores interessados no processo de TD (VIAL, 2019). Nesse sentido, engajamentos com startups são uma forma de inovação aberta cada vez mais 
considerada para se catalisar o processo de digitalização de empresas estabelecidas (WEIBLEN; Chesbrough, 2015; BAgno et al., 2020; NADKARNi; PrÜGL, 2020).

\subsection{Processo de Inovação como condutor de projetos associados à Transformação Digital}

Um Sistema de Gestão da Inovação tem no processo de inovação seu elemento mais central. Por meio do estabelecimento de um processo de inovação que se pavimenta a dinâmica típica de identificação de novas ideias, sua seleção / priorização e o desenvolvimento destas até se consolidarem como inovações difundidas no mercado (ou para clientes internos, conforme o caso) (BAgno; SAlERno; Silva, 2017). A centralidade do processo de inovação como elemento organizador dos esforços sistemáticos de inovação de uma empresa ganhou na literatura uma perspectiva destacada a ponto de se negligenciar o papel de outros elementos necessários ao sistema em muitos dos estudos realizados (O'CONNOR et al., 2008; BAGNO; SALERNO; SILVA, 2017). Associado ao processo de inovação, um SGI deve ser delineado para estabelecer uma dinâmica de gestão de portfólio de projetos que esteja adaptada para lidar com oportunidades de alta incerteza e que identifique adequadamente recursos complementares necessários para alavancar iniciativas de alto valor estratégico (O'CONNOR et $a l .$, 2008; BAGNO; SALERnO; Silva, 2017). Tal abordagem é aderente às ações e iniciativas ligados à TD se geridas como projetos em um portfólio de inovação.

\subsection{Transformação Digital como propulsor do Sistema de Gestão da Inovação}

Se, por um lado, o aparato gerencial dos SGI parece ser capaz de acomodar e amoldar-se com robustez a demandas das iniciativas de TD, na via inversa a TD pode ser vista como um propulsor dos SGI sob ao menos duas perspectivas.

Primeiramente, a digitalização dos processos de inovação e suas adjacências traz maior dinamicidade e integração entre suas diferentes fases. Por exemplo, de acordo com Ries (2011) as novas infraestruturas digitais, como impressão 3D e marketplaces, permitem que ideias de produtos sejam rapidamente formadas, aprovadas, modificadas e reencenadas por meio de ciclos repetidos de experimentação e implementação. Da mesma forma, tecnologias como computação em nuvem, Big Data e aprendizado de máquina na identificação de oportunidades de negócios ou novas ferramentas colaborativas e virtuais para times de inovação permitem atingir novos novo níveis de fluidez nos processos de inovação, permitindo que projetos se desdobrem de uma forma menos linear e mais ágil e que o conhecimento e envolvimento de colaboradores e parceiros externos se consolide de uma forma não antes testemunhada. Como observado por Boland; Lyytinen e Yoo (2007) o uso de 
ferramentas 3D como uma infraestrutura de processo digital em inovações na construção levou a interações e colaborações inesperadas entre diferentes ofícios, designers e outras partes interessadas, gerando múltiplos “despertares de inovação”.

Em segundo lugar, é necessário observar que a revolução digital é um fenômeno de natureza social e, como tal, está associado a diversas transformações de valores, comportamentos e estilos de vida que tem impactado diretamente a forma como pensamos o trabalho dentro de organizações empresariais. Tendências como adoção de equipes ágeis cada vez mais autônomas nas linhas de frente e intrinsecamente motivadas pelos propósitos amplos de seus projetos, relações interorganizacionais mais horizontais e flexíveis baseadas mais fortemente em confiança e ganhos mútuos do que na formalidade dos contratos, a proeminência da figura do empreendedor no contexto empresarial, o contato mais direto e de caráter colaborativo com clientes e outros stakeholders, o desenvolvimento de inovações por meio de contínua experimentação, além de uma valorização crescente de transparência e compromisso de facto com transformações socioambientais para além da visão estrita do negócio das empresas são ingredientes notórios dessa revolução. Como tais, não somente trazem impactos significativos nas formas de operação de sistemas gestão e de seus métodos e ferramentas associadas, mas também à própria forma como temos pensado a natureza e operação das organizações contemporâneas.

\section{CONCLUSÃO}

Sistemas de Gestão da Inovação e Transformação Digital tem seguido trajetórias paralelas no mainstream de pesquisa, ao passo que há grandes oportunidades no desenvolvimento de uma visão integrada entre estes temas. Esse estudo relata uma caminhada inicial nesta reflexão, trazendo para discussão os pontos mais imediatos.

Como continuidade do estudo, o desafio a ser priorizado está no redesenho de um modelo de referência para sistemas de gestão da inovação ajustando-o às demandas, mas também às novas potencialidades do contexto da transformação digital. Entende-se que o adequado embora complexo - equilíbrio entre disciplina e flexibilidade associado à concatenação dos elementos propostos por diferentes vertentes no debate da TD e a escola mais consolidada dos SGI constituem solo fértil para a proposição de abordagens que auxiliem empresas de diferentes portes e setores a constituírem capacidades de inovação digital que pavimentem sua trajetória neste novo contexto. 


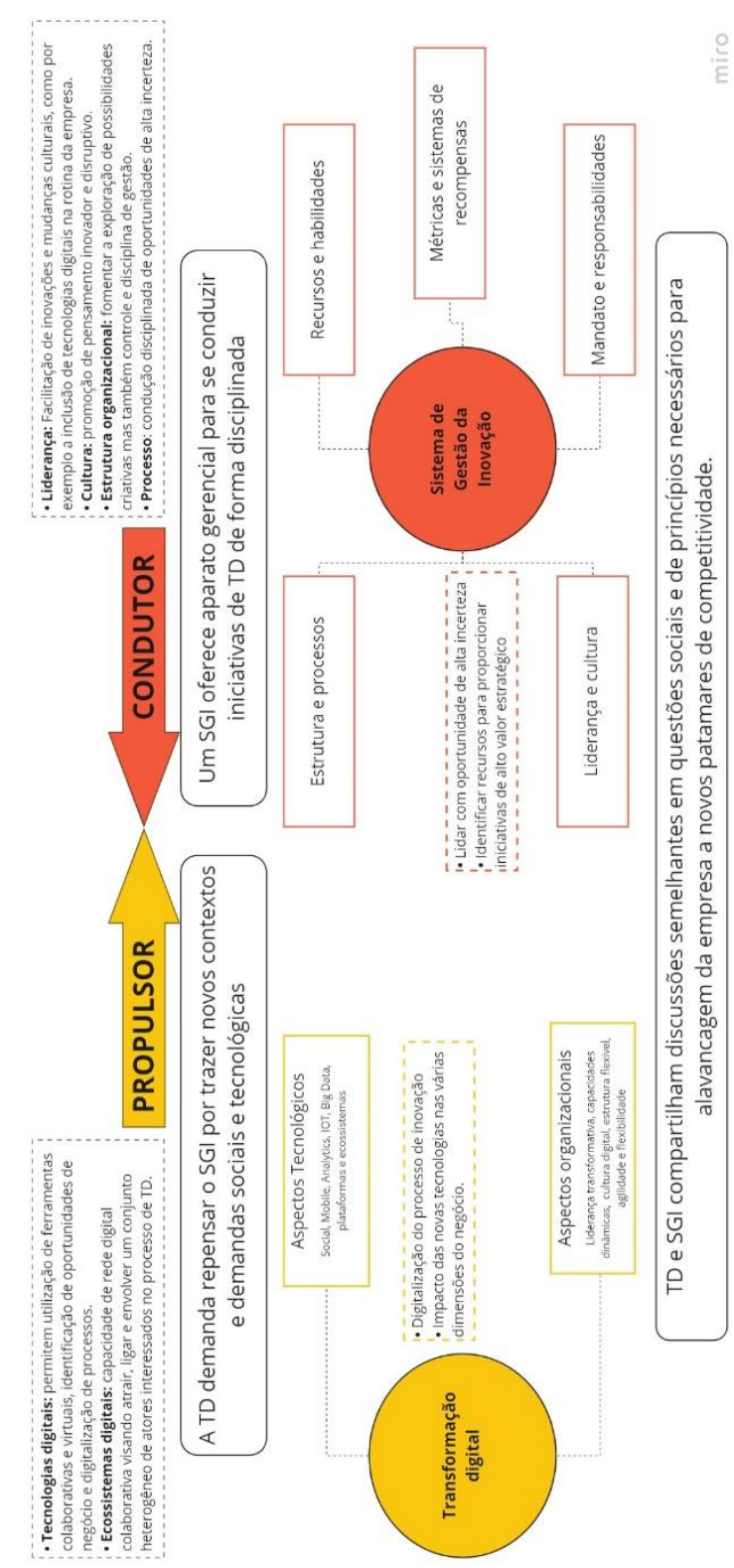

Figura 4 - Proposta de integração entre SGI e TD. Fonte: dos autores.

\section{REFERÊNCIAS}

ADAMS, R.; BESSANT, J.; PHELPS, R. Innovation management measurement: A review. International Journal of Management Reviews, v. 8, n. 1, p. 21-47, 2006.

ANTHONY, S.D.; JOHNSON, M.W.; SINFIELD, J.V.J.M.S.M.R. Institutionalizing innovation. MIT Sloan Management Review, v. 49, n. 2, p. 45, 2008.

BAGNO, R.B.; LEIVA, T.L.; OLIVEIRA, L.G.H. Innovation management: lessons learned from innovation diagnostic tools. Product: Management and Development, v. 14, n. 1, p. 12-21, 2015.

BAGNO, R.B.; SALERNO, M.S.; SILVA, D.O. Models with graphical representation for innovation management: a literature review. R\&D Management, v. 47, n. 4, p. 637-653, 2017.

BAGNO, R.B.; SALERNO, M.S.; SOUZA JUNIOR, W.C.; O'CONNOR, G.C. Corporate engagements with startups: antecedents, models, and open questions for innovation management. Product: Management and Development, v. 18, n. 1, p. 39-52, 2020. 
BOLAND, R.J.; LYYTINEN, K.; YOO, Y. Wakes of Innovation in Project Networks: The Case of Digital 3-D Representations in Architecture, Engineering, and Construction. Organization Science, v. 18, n. 4, p. 631-647, 2007.

BURRI, M. Current and Emerging Trends in Disruptive Technologies: Implications for the Present and Future of EU's Trade Policy. Study for the European Parliament, v. 603, 10/01 2017.

BUVAT, J.; CRUMMENERL, C.; KAR, K.; SENGUPATA, A.; SOLIS, B.; ABOUD, C.; AL AOUFI, $\mathrm{H}$. The digital culture challenge: Closing the employee-leadership gap. Hg. v. Capgemini (Abruf 14.03. 2019). 2017

CROSSAN, M.M.; APAYDIN, M. A Multi-Dimensional Framework of Organizational Innovation: A Systematic Review of the Literature. Journal of Management Studies, v. 47, n. 6, p. 1154-1191, 2010.

DENNING, S. The age of agile : how smart companies are transforming the way work gets done. Amacom, 2018.

ERNST, H. Success Factors of New Product Development: A Review of the Empirical Literature. International Journal of Management Reviews, v. 4, n. 1, p. 1-40, 2003.

FRAMBACH, R.T.; SCHILLEWAERT, N. Organizational innovation adoption: A multi-level framework of determinants and opportunities for future research. Journal of business research, v. 55, n. 2, p. 163-176, 2002.

GEBAYEW, C.; HARDINI, I.R.; PANJAITAN, G.H.A.; KURNIAWAN, N.B.; SUHARDI; IEEE. A Systematic Literature Review on Digital Transformation. In: SUHARDI;LANGI, A. Z. R., et al (Ed.). 2018 International Conference on Information Technology Systems and Innovation. New York: Ieee, 2018. p.260-265. (International Conference on Information Technology Systems and Innovation (ICITSI)).

HANELT, A.; BOHNSACK, R.; MARZ, D.; ANTUNES MARANTE, C. A systematic review of the literature on digital transformation: insights and implications for strategy and organizational change. Journal of Management Studies, 2020.

HANSEN, M.T.; BIRKINSHAW, J. The innovation value chain. Harvard Business Review, v. 85, n. 6, p. 121, 2007.

KOPALLE, P.K.; KUMAR, V.; SUBRAMANIAM, M. How legacy firms can embrace the digital ecosystem via digital customer orientation. Journal of the Academy of Marketing Science, v. 48, n. 1, p. 114-131, 2020.

MIR, M.; CASADESÚS, M.; PETNJI, L.H. The impact of standardized innovation management systems on innovation capability and business performance: An empirical study. Journal of Engineering and Technology Management, v. 41, p. 26-44, 2016/07/01/ 2016.

MUMFORD, M.D.; LICUANAN, B. Leading for innovation: Conclusions, issues, and directions. The Leadership Quarterly, v. 15, n. 1, p. 163-171, 2004/02/01/ 2004.

NADKARNI, S.; PRÜGL, R. Digital transformation: a review, synthesis and opportunities for future research. Management Review Quarterly, p. 1-109, 2020.

NAMBISAN, S.; LYYTINEN, K.; MAJCHRZAK, A.; SONG, M. Digital Innovation Management: Reinventing Innovation Management Research in a Digital World. MIS Quarterly, v. 41, 01/01 2017.

O'CONNOR, G.; LEIFER, R.; PAULSON, A.; PETERS, L. Grabbing Lightning: Building a Capability for Breakthrough Innovation. John Wiley \& Sons, 2008.

RIASANOW, T.; SETZKE, D.S.; BÖHM, M.; KRCMAR, H. Clarifying the notion of digital transformation: a transdisciplinary review of literature. Journal of Competences, Strategy and Management, v. 10, p. 5-31, 2019. 
RIES, E. The lean startup: How today's entrepreneurs use continuous innovation to create radically successful businesses. Currency, 2011.

ROMERO, D.; FLORES, M.; HERRERA, M.; RESENDEZ, H. Five Management Pillars for Digital Transformation Integrating the Lean Thinking Philosophy. In: (Ed.). 2019 IEEE International Conference on Engineering, Technology and Innovation (ICE/ITMC), 2019. p.1-8.

SEBASTIAN, I.; ROSS, J.; BEATH, C.; MOCKER, M.; MOLONEY, K.; FONSTAD, N. How big old companies navigate digital transformation. MIS Quarterly Executive, v. 16, n. 3, p. 197-213, 2017.

TEECE, D.J.; PISANO, G.; SHUEN, A. Dynamic capabilities and strategic management. Strategic management journal, v. 18, n. 7, p. 509-533, 1997.

TIDD, J. A Review And Critical Assessment Of The Iso56002 Innovation Management Systems Standard: Evidence And Limitations. International Journal of Innovation Management, p. 2150049, 2021.

VAN LANCKER, J.; MONDELAERS, K.; WAUTERS, E.; VAN HUYLENBROECK, G. The Organizational Innovation System: A systemic framework for radical innovation at the organizational level. Technovation, v. 52-53, p. 40-50, 2016/06/01/ 2016.

VERHOEF, P.C.; BROEKHUIZEN, T.; BART, Y.; BHATTACHARYA, A.; DONG, J.Q.; FABIAN, N.; HAENLEIN, M. Digital transformation: A multidisciplinary reflection and research agenda. Journal of Business Research, v. 122, p. 889-901, Jan 2021.

VIAL, G. Understanding digital transformation: A review and a research agenda. The Journal of Strategic Information Systems, v. 28, n. 2, p. 118-144, 2019.

WEIBLEN, T.; CHESBROUGH, H.W. Engaging with startups to enhance corporate innovation. California management review, v. 57, n. 2, p. 66-90, 2015. 